\title{
Maternal and child health indicators: implications of the tenth revision of the International Classification of Diseases ${ }^{1}$
}

\author{
Ruy Laurenti² and Cássia Maria Buchalla²
}

\begin{abstract}
Since the end of the 1940s, when the World Health Organization assumed responsibility for the decennial revisions of the Classification of Causes of Death, the Classification came to include diseases and definitions of use in vital statistics, resulting in the Sixth Revision of the International Classification of Diseases (ICD-6). The most recent revision of this work, the International Statistical Classification of Diseases and Related Health Problems (ICD10), is more significantly different than any revision since ICD-6, especially in the area of maternal and child health. Among the changes introduced in ICD-10 are the inclusion of obstetrical tetanus in the chapter on infectious diseases, which will facilitate the recording of this cause of maternal death; the incorporation of new definitions, such as late maternal death; and the redefinition of the perinatal period, which ICD-10 defines as starting at 22 completed weeks of gestation and ending 7 completed days after birth. This article seeks to highlight these changes and to discuss their consequences for the presentation and interpretation of indicators used in the evaluation of maternal and child health.
\end{abstract}

In the latter half of the 1940s, the World Health Organization (WHO) assumed responsibility for the decennial revisions of the Classification of Causes of Death, also known as Bertillon's Classification. The first revision affected was the sixth, which was approved in 1948 and put into use in 1950. Beginning with that revision, the classification came to include diseases

\footnotetext{
1 A Portuguese version of this article has been published in this journal (Vol 1, No 1, 1997, pp. 18-23) with the title "Indicadores da saúde materna e infantil: impliçacões da décima revisão da Classificação Internacional de Doenças."

2 World Health Organization Collaborating Center for the Classification of Diseases in Portuguese, Department of Epidemiology, School of Public Health, University of São Paulo, São Paulo, Brazil. Mailing address: Av. Dr. Arnaldo, 715, CEP 01246904, São Paulo, SP, Brazil.
}

other than those causing death and made the important change of incorporating definitions of use in vital statistics. In addition, following the sixth revision the classification itself was expanded. At present, the term "Classification of Diseases" encompasses what is in fact a family of classifications, as well as definitions and recommendations of use in health statistics, particularly vital statistics $(1,2)$.

In the area of maternal and child health, the International Classification of Diseases (ICD) includes definitions of live birth, fetal death, infant death, maternal death, perinatal death, and other terms, as well as formulas for calculating the respective rates involved and recommendations for making statistical presentations. The latest revision, ICD-10 (3), incorporates more changes from the preceding revision than any revision since ICD-6. These changes consist of modifications and (primarily) expansions throughout the text, including those chapters relating to maternal and child health. It is important to point out that a number of definitions were modified. It should also be noted that the title of the ICD itself was changed to International Statistical Classification of Diseases and Related Health Problems, reflecting the work's greater scope. The purpose of the article presented here is to highlight the changes introduced in ICD-10 and to discuss their consequences for the presentation and interpretation of indicators used in the evaluation of maternal and child health. 


\section{CHAPTERS RELATING TO MATERNAL AND CHILD HEALTH}

With regard to maternal health, and more especially to problems of pregnancy, childbirth, and the puerperium, ICD-6 and its successors have included a chapter entitled "Complications of Pregnancy, Childbirth, and the Puerperium," a title shortened in ICD-10 to "Pregnancy, Childbirth, and the Puerperium." With regard to newborn health, ICD-10 contains a chapter covering disorders occurring in the perinatal period. However, disorders classified in other chapters, primarily those involving congenital malformations, may be used in describing the frequency of these disorders in studies of perinatal morbidity and mortality.

\section{Chapter XV: Pregnancy, childbirth, and the puerperium (O00-O99)}

This chapter currently includes eight groupings (compared to five groupings in ICD-9). The number of categories has also increased, from 45 (ICD-9) to 75 (ICD-10). Although the structure of the chapter has not changed, the modifications introduced will facilitate its use in determining indexes of both morbidity and mortality. It should be noted the ICD-10 chapter includes 25 "blank" categories, i.e., categories with no disorders allocated, which allows users to make expansions for the purpose of achieving greater specificity, particularly in the area of morbidity. The corresponding ICD-9 chapter had but one blank category.

More important, the content of the chapter has been made more logical and satisfactory for users in the maternal health field, including workers in both obstetrics and public health. For example, prior to ICD-9 it was difficult or impossible to classify a maternal disorder (causing either morbidity or mortality) when it was not precisely known whether the complication involved pregnancy, childbirth, or the puerperium. ICD-10 deals with this matter, as will be discussed later.
Another key change is inclusion of the category "Obstetrical Tetanus" in the chapter dealing with infectious diseases. In ICD-9, this category was not identifiable as tetanus. Obstetric tetanus was included in the chapter on complications occurring during pregnancy, childbirth, or the puerperiumalong with other infectious diseases that may follow abortion, ectopic or molar pregnancy, and the puerperium. This change is relevant in view of the magnitude of the maternal mortality problem in Latin America (4, $5)$. Thus, the opportunity to characterize obstetric tetanus in maternal mortality statistics (something that was not possible with ICD-9) should be of considerable significance in diagnosing situations and implementing specific preventive actions. However, it is important to exercise caution when calculating maternal health mortality statistics, since it is now necessary to add deaths classified as due to "obstetrical tetanus" in Chapter I (which deals with infectious and parasitic diseases) to those due to the other causes of maternal mortality classified in Chapter XV.

ICD-10 also introduces three longawaited categories for use in conducting analyses of maternal mortality that will make it possible to recognize maternal mortality in situations where it could not previously be identified. The first of these categories is "Obstetric Death of Unspecified Cause," a category occurring frequently in descriptive sampling (in survey-type research efforts) conducted for the purpose of measuring maternal deaths. The second new category, discussed in the section below dealing with definitions, is "Death from Any Obstetric Cause Occurring More Than 42 Days But Less Than One Year after Delivery." The third category, "Death from Sequelae of Direct Obstetric Causes," makes it possible to codify (and accordingly identify) deaths from direct obstetric causes occurring more than one year after delivery. That category is significant because recent therapeutic advances have led to longer survival times for the victims of com- plications of pregnancy, childbirth, and the puerperium-so that death from such causes can indeed occur after 42 days, and it is not uncommon for it to occur as long as one year after delivery.

\section{Chapter XVI: Certain conditions originating in the perinatal period (P00-P99)}

This chapter has its origins in the ICD-6 and ICD-7 chapters entitled "Diseases Peculiar to Early Infancy." In ICD-8, the chapter title changed to "Certain Causes of Perinatal Morbidity and Mortality" and, finally, in ICD-9 and ICD-10, the title changed again (and more appropriately) to "Certain Conditions Originating in the Perinatal Period." The qualifier "originating" means that the categories in this chapter can be used in studies of morbidity and mortality in the perinatal period and also in cases where death occurred after that period. In fact, this qualifier is frequently used during the neonatal and infant periods, and it is not uncommon for it to be used after one year of age.

The body of Chapter XVI also underwent significant changes from ICD-9 to ICD-10. For one thing it was expanded-from 20 categories in ICD-9 to 58 (organized in 10 groupings) in ICD-10. In response to the importance of perinatal mortality (primarily in developed countries, where it accounts for the bulk of infant mortality) the expansion of this chapter facilitates characterization and specification of the causes of perinatal mortality.

Several groupings in the chapter seem especially deserving of mention. The categories subsumed under the grouping labeled "Disorders Related to Length of Gestation and Fetal Growth" (P05-P08) present glossarytype definitions. Another important grouping, particularly for developing countries, is the one referring to "Infections Specific to the Perinatal Period" (P35-P39). Despite some exceptions, this grouping deals with 
infectious or parasitic diseases that are either congenital or acquired during childbirth. An important change in this grouping was the exclusion of neonatal tetanus, which in ICD-10 is included in the chapter on infectious and parasitic diseases (category A33). In ICD-9, neonatal tetanus was a subcategory, and so it did not routinely appear in tabulations as a cause of neonatal or infant death. (Congenitally transmitted AIDS, the incidence of which is increasing in many countries, is also included in the chapter dealing with infectious and parasitic diseases.) Expansion of the grouping "Hemorrhagic and Hematological Disorders of Fetus and Newborn" (P50-P61) from two categories in ICD-9 to 12 in ICD-10 should facilitate more precise specification of the pathologies described in that grouping - a matter of great interest, particularly for morbidity studies.

\section{Other chapters of interest}

The causes linked to characterization of maternal morbidity and mortality, with the exception of obstetric tetanus, are presented in Chapter XV. The classification of causes of morbidity or mortality occurring or originating in the perinatal, neonatal, and infant periods is based on the categories dealing with complications in the perinatal period and those in Chapter XVII, "Congenital Malformations, Deformations, and Chromosomal Abnormalities" (Q00-Q99). These latter play a large role primarily in areas where infant mortality is low and concentrated in the neonatal period, particularly the early neonatal period. However, the categories included in the chapters dealing with perinatal causes and congenital malformations are also used a great deal in developing countries for elaborating morbidity and mortality statistics relating to causes originating in the neonatal and infant periods. Morbidity and mortality patterns in these countries also involve a large number of causes classified in the chapter on infectious and parasitic diseases and the chapter on respiratory diseases.

The chapter dealing with congenital malformations in ICD-10 (based largely on recommendations of the British Pediatric Association and the International Clearinghouse on Birth Defects Monitoring System) affords a considerable degree of specificity, as reflected in its inclusion of 87 categories (compared to 20 in ICD-9).

\section{MATERNAL AND CHILD HEALTH DEFINITIONS}

As already noted, the ICD includes definitions of interest for health statistics, particularly in the area of vital statistics. Some of these definitions were modified in ICD-10, and new definitions were introduced. Epidemiologists and health service administrators need to be aware of these changes when using indicators to help analyze health situations or evaluate maternal and child health programs. Definitions for use in health statistics can be found in the ICD instruction manual (6).

\section{Maternal deaths}

While ICD-10 was being prepared, numerous requests were received to change the definition of maternal death (which appears for the first time in ICD-9) by extending the period of maternal death from up to 42 days after childbirth to up to one year after childbirth. This change was not made for a number of reasons, including worry that studies of trends would be affected. However, as noted in the section on Chapter $\mathrm{XV}$, a new category was included in that chapter to cover "late" maternal death from any obstetric cause occurring more than 42 days but less than one year after childbirth.

ICD-10 recommends that maternal mortality rates and ratios continue to be calculated on the basis of the current definition, which limits this period to 42 days following childbirth. Those wishing to calculate a second rate including late maternal deaths should take care to clearly distinguish between the two rates in their presentations. In addition, the ICD-10 instruction manual recommends that death certificates include matters related to pregnancy in process at the time of death or pregnancy occurring during the year preceding death (6). That recommendation is aimed at improving the quality of maternal mortality data, strengthening alternative methods for collecting data on death during pregnancy, and encouraging the recording of deaths from obstetric causes occurring more than 42 days after termination of pregnancy. With regard to the causes of maternal death, ICD-10 retains the previously existing arrangement for classifying such deaths into one of two groups: direct and indirect obstetric causes.

Expressing another new concept, ICD-10 defines "pregnancy-related death" as death during pregnancy or within 42 days of the termination of pregnancy, regardless of the cause of death. ICD-10 also proposes calculating a ratio for pregnancy-related mortality in which the numerator represents maternal deaths from direct and indirect obstetric causes plus deaths attributed to any other cause (nonobstetric). It is clear that this ratio can be calculated only in countries where information recorded on the death certificate includes information about pregnancy in progress at the time of death or pregnancy occurring during the year preceding death. Since the causes of maternal death are generally not clearly indicated on death certificates, it is possible to conclude that the new definition, as well as calculation of the ratio, is directed at capturing cases of maternal death not characterized as such.

ICD-10 also contains definitions of ratios and rates related to maternal mortality. This section recommends that care be taken to include maternal deaths caused by the human immunodeficiency virus (HIV) (B20-B24) and obstetric tetanus in the maternal mortality ratio, even though these causes are codified in categories found in other chapters. 
Fetal, perinatal, neonatal, and infant mortality

Aside from a change in the definition of the perinatal period, which will be discussed below, the definitions of "live birth," "fetal death," "birthweight," "low birthweight," "gestational age," "pre-term," and "postterm" were not modified in ICD-10. However, definitions relating to fetal mortality rates and ratios and neonatal and infant mortality rates were modified.

\section{The perinatal period and perinatal mortality}

Definition of the perinatal period was modified in ICD-10. Consequently, interpretation of the term "perinatal death" and respective mortality rates must also be changed. Since perinatal mortality is an indicator used increasingly to measure infant health and is also recommended for evaluating maternal health, a more extensive discussion of the reasons for this change seems in order.

Peller (7) proposed the term "perinatal" in 1940, as he considered it necessary to analyze as a "statistical unit" the total number of stillbirths (late fetal losses) in conjunction with deaths occurring during the first seven days following delivery. For statisticians, the use of that concept facilitated comparisons of data at the international level.

Clinicians and epidemiologists will perceive that the two components of perinatal mortality are subject to the same prenatal and intranatal causative factors. However, the concept of the perinatal period and deaths occurring during that period, as proposed by Peller, was not always correctly applied, especially with regard to what was known as "stillbirth." Indeed, the concept of stillbirth varied from country to country, and the prevailing legislation did not always define the term or make its recording mandatory. After assuming responsibility for suc- cessive revisions of the International Classification of Diseases, WHO included in ICD-7 (1955) the definitions of "live birth" and "fetal death," facilitating an international consensus on "stillbirth" as being synonymous with "late fetal death" or death occurring after at least 28 weeks of gestation (8). ICD-7 also provided for recording and tabulating fetal deaths as having occurred in "early," "intermediate," or "late" periods in accordance with the same periods of gestation applied to live births. The term abortion was not defined.

ICD-7 recommended that the term "stillbirth" be used as a synonym for "late fetal death," and so it could be utilized this way by countries elaborating statistics on stillbirths.

ICD-8 defined the perinatal period as extending from the beginning of the 28th week of gestation up to the seventh day of life (9). Thus, the term perinatal and its respective concept, as proposed by Peller (7), came to be used internationally. Subsequently, ICD-9 included other elements to characterize the beginning of the perinatal period, using weight (1 $000 \mathrm{~g}$ or more) as the principal defining factor, followed by corresponding gestational age (at least 28 weeks) when birthweight was not available, or birth length (at least $35 \mathrm{~cm}$, measured from the top of the head to the heel) (10). In the 1970s, however, neonatologists and obstetricians had already concluded that the perinatal period should be defined as beginning before the 28th week of pregnancy; for this reason, ICD-9 encouraged national perinatal statistics to include all fetuses and newborns with a minimum weight of $500 \mathrm{~g}$, which corresponds to a gestational age of 22 weeks. For purposes of international comparisons, the limit continued to be $1000 \mathrm{~g}$ or a minimum of 28 weeks of gestation.

In ICD-10, the perinatal period is defined as beginning at 22 full weeks (154 days) of gestation, when birthweight is normally $500 \mathrm{~g}$, and ending seven full days after delivery. This change will have consequences for statistics involving stillbirths and perinatal mortality. Beginning in the 22nd week, fetal losses will be added to the numerator in calculating perinatal mortality. Accordingly, perinatal mortality will increase, with corresponding consequences for trend studies.

Some scientific publications are already taking this change into account (11-14). The term "stillborn" will probably now be identified with fetal losses beginning at the 22nd week of gestation, or when the fetus attains a weight of $500 \mathrm{~g}$. These losses will also probably be known as "late fetal losses." Thus, a new way to calculate the rate of stillbirths will be available, providing a useful indicator for evaluating maternal health care.

Within this context, it is important to note an issue related to the international comparability of stillbirths and perinatal mortality. Legislation varies from one country to another with regard to the requirement that fetal losses be recorded in the civil registry. In some countries there are legal requirements for recording all fetal losses; in others only losses occurring as of the 20th week must be recorded; and in others only the recording of stillbirths is required. In the latter cases, it is common for stillbirths to be considered synonymous with late fetal losses, these being defined as beginning at the 28th week of gestation. Since it is difficult to change legislation, it is possible to foresee difficulties in calculating stillbirths and perinatal mortality based on the new definition of the perinatal period included in ICD-10. This matter must be taken into consideration by epidemiologists and health administrators working in the field of maternal and child health. In the Region of the Americas, where $\mathrm{PAHO}$ is implementing a project aimed at reducing maternal mortality (5), it will be necessary to discuss this problem and propose solutions, including possible changes to legislation, since these indicators are extremely useful for evaluating programs in the area of maternal and child health. 


\section{REFERENCES}

1. Laurenti R. Análise da informação em saúde: 1893-1993; cem anos da classificação internacional de doenças. Rev Saude Publica 1991; 25(6):407-417.

2. Laurenti R. Décima revisão da classificação internacional de doenças e de problemas relacionados à saúde (CID-10): a revisão do final do século. Bol Oficina Sanit Panam 1995;118(3): 273-280.

3. World Health Organization. Volume 1: International statistical classification of diseases and related health problems: tenth revision. Geneva: WHO; 1992.

4. Laurenti R. Maternal mortality in Latin American urban areas: the case of São Paulo, Brazil. Bull Pan Am Health Organ 1993;27(3): 205-214.

5. Pan American Health Organization. Regional plan of action for reduction of maternal mortality in the Americas. Washington, DC: PAHO; 1990. (Document CSP23/10).
6. World Health Organization. Volume 2: International statistical classification of diseases and related health problems: tenth revision; instruction manual. Geneva: WHO; 1992

7. Peller S. Proper delineation of the neonatal period in perinatal mortality. Am J Public Health 1965;55:1005-1011.

8. World Health Organization. Manual of the international statistical classification of diseases, injuries, and causes of death: seventh revision. Geneva: WHO; 1957.

9. World Health Organization. Manual of the international statistical classification of diseases, injuries, and causes of death: eighth revision. Geneva: WHO; 1967.

10. World Health Organization. Manual of the international statistical classification of diseases, injuries, and causes of death: ninth revision. Geneva: WHO; 1977.

11. Wigglesworth JS. Classification of perinatal deaths. Soz Praventivmed 1994;39:11-14.
12. Gissler M, Ollila E, Teperi J, Hemminki E. Impact of induced abortions and statistical definitions on perinatal figures. Paediatr Perinat Epidemiol 1994;8:391-400.

13. Gourbin C, Masuy-Stroobant G. Are live and stillbirths comparable all over Europe? Legal definitions and vital registration data processing. International Institute for Vital Registration and Statistics; August 1994. [Technical Paper 58.]

14. Cartlidge $\mathrm{PH}$, Steward JH. Effect of changing the stillbirth definition on evaluation of perinatal mortality rates. Lancet 1995;346:486-488.

Manuscript received on 30 November 1995. Revised version accepted for publication on 4 June 1996.

RESUMEN A partir de finales de los años cuarenta, cuando la OMS asumió la responsabilidad de revisar cada decenio la Clasificación Internacional de Causas de Defunción, la Clasificación vino a incluir enfermedades y definiciones de utilidad para

Indicadores de salud materna e infantil: implicaciones de la décima revisión de la Clasificación Internacional de Enfermedades las estadísticas vitales, y ello dio por resultado la Sexta Clasificación Internacional de Enfermedades (CID-6). La revisión más reciente de esta obra, que es la Clasificación Estadística Internacional de Enfermedades y Problemas Relacionados con la Salud (CID-10), representa los cambios más importantes que se han efectuado desde la CID-6, principalmente en lo relativo al área maternoinfantil. Entre los cambios introducidos en la CID-10 figuran la inclusión del tétanos obstétrico en el capítulo sobre enfermedades infecciosas, que facilitará el registro de esta causa de muerte materna; la incorporación de nuevas definiciones, tales como la de muerte materna tardía; y la redefinición del período perinatal, que según lo define la CID-10 se extiende desde las 22 semanas de gestación hasta el séptimo día después del nacimiento. El artículo resalta estos cambios y examina sus consecuencias para la presentación e interpretación de los indicadores que se usan en la evaluación de la salud materna e infantil. 